FACTA UNIVERSITATIS (NIŠ)

Ser. Math. Inform. Vol. 34, No 5 (2019), 871-888

https://doi.org/10.22190/FUMI1905871A

\title{
MORE ON INTUITIONISTIC FUZZY SUBLATTICES AND THEIR IDEALS
}

\author{
Abdelaziz Amroune and Brahim Ziane \\ (C) 2019 by University of Niš, Serbia | Creative Commons Licence: CC BY-NC-ND \\ Abstract. In this paper, we study the concept of intuitionistic fuzzy sublattices and \\ intuitionistic fuzzy ideals with respect to an intuitionistic fuzzy t-norm on an adequate \\ lattice. Some characterizations and properties of these intuitionistic fuzzy sublattices \\ and ideals with respect to intuitionistic fuzzy t-norm are established. \\ Keywords. Intuitionistic fuzzy sublattices; intuitionistic fuzzy ideals; intuitionistic \\ fuzzy t-norm.
}

\section{Introduction}

In 1965, Zadeh introduced the concept of fuzzy sets (FS for short) establishing its fundamental properties [24]. Later, several authors studied this new concept and its applications to many areas of modern mathematics, see for example [11-13,15]. In the same way, Yuan and $\mathrm{Wu}[6]$ studied the relationship between fuzzy ideals and fuzzy congruences on a distributive lattice and obtained that the lattice of fuzzy ideals is isomorphic to the lattice of fuzzy congruence on a generalized Boolean algebra. Ajmal and Thomas [2] defined and characterized fuzzy sublattice as a fuzzy algebra. In 1983, Atanassov [4] introduced the concept of intuitionistic fuzzy sets (IFS for short) and established many of its fundamental properties. After a few years, Thomas and Nair $[22,23]$ studied intuitionistic fuzzy sublattice, intuitionistic fuzzy ideals and intuitionistic fuzzy filters on a lattice, for more details we refer to $[1,14,16,17,20,22,23]$. In this paper, we introduce the notion of $\mathcal{T}$-intuitionistic fuzzy sublattice by associating the conditions mentioned in the definition of an intuitionistic fuzzy sublattice $[22,23]$ we obtain a general condition which reduces the four conditions in only one. Thus, based on an intuitionistic fuzzy triangular norm, the study of intuitionistic fuzzy sublattice becomes so simple. Moreover, we extend the notion of intuitionistic fuzzy ideal to $\mathcal{T}$-intuitionistic fuzzy ideal with

Received October 10, 2018; accepted November 24, 2019

2010 Mathematics Subject Classification. Primary 03F55; Secondary 03E72, 06D72 
respect to ( $\mathcal{T}$-intuitionistic fuzzy ideal w.r.t for short) the lattice operations and we investigate their various characterizations and properties.

The remainder of the paper is structured as follows. In Section 2, we recall the necessary basic concepts and properties of fuzzy sublattices and fuzzy ideals, intuitionistic fuzzy set. Also, we recall some definitions and theorems which we will need in the sequel.

In Section 3, we introduce the notion of intuitionistic fuzzy Triangular norms and Triangular conorms as well as some concepts related to an intuitionistic fuzzy triangular norm such as triangular intuitionistic fuzzy sublattices and the intersection of triangular intuitionistic fuzzy subsets w.r.t an intuitionistic triangular norm. In Section 4, we introduce and study the notion of triangular intuitionistic fuzzy ideals and we provide several characterizations results related to triangular intuitionistic fuzzy sublattices, triangular intuitionistic fuzzy subsets, and triangular intuitionistic fuzzy ideals. Finally, some conclusions and open questions are given in Section 5.

\section{Preliminaries}

The purpose of this section is to provide a basic introduction to the poset, lattices and ideal and we recall some definitions and theorems which we need in the sequel. Throughout this paper, $L$ denotes a lattice $(L, \leqslant)$ and $L^{d}$ denotes its dualorder lattice $(L, \geqslant)$. To avoid any confusion or misunderstanding in some formulas, we use the notation $(\leqslant, \wedge, \vee)$ to refer the (order, min, max) on the lattice $L$ and $(\leq, \min , \max )$ to refer the (usual order, $\min , \max )$ on the real interval $[0,1]$.

\subsection{Fuzzy Sublattices}

According to Zadeh [24], a fuzzy set on a set $X$ is defined to be a mapping :

$$
\mu: X \rightarrow[0,1]
$$

For $x \in X, \mu(x)$ is interpreted as the degree of membership of $x$ in the fuzzy set $\mu$.

Definition 2.1. [23] A fuzzy subset $\mu$ of $L$ is called a fuzzy sublattice of $L$ if,

(i) $\mu(x \vee y) \geq \min (\mu(x), \mu(y)\}$

(ii) $\mu(x \wedge y) \geq \min (\mu(x), \mu(y)\}, \forall x, y \in L$.

Definition 2.2. [23] A fuzzy subset $\mu$ of $L$ is called a fuzzy ideal of $L$ if,

(i) $\mu(x \vee y) \geq \min (\mu(x), \mu(y)\}$

(ii) $\mu(x \wedge y) \geq \max (\mu(x), \mu(y)\}, \forall x, y, \in L$. 


\subsection{Intuitionistic fuzzy sets}

The definition of an intuitionistic fuzzy set was introduced by Atanassov $[4,5]$.

Definition 2.3. [4] Let $X$ be a non-empty set. An intuitionistic fuzzy set (IFS) $A$ of $X$ is an object of the following form $A=\left\{\left(x, \mu_{A}(x), \nu_{A}(x)\right) \mid x \in X\right\}$, where $\mu_{A}: X \rightarrow[0,1]$ and $\nu_{A}: X \rightarrow[0,1]$ define the degree of membership and degree of nonmembership of the element $x \in X$ respectively and for any $x \in X$, we have $0 \leq \mu_{A}(x)+\nu_{A}(x) \leq 1$. The collections of all intuitionistic fuzzy set of $X$ will be denoted by $\operatorname{IFS}(X)$.

Note that for the extreme case $\mu_{A}(x)+\nu_{A}(x)=1, A$ is a fuzzy set.

Definition 2.4. $\quad[22,23]$ Let $L$ be a lattice and $A=\left\{x, \mu_{A}(x), \nu_{A}(x) / x \in L\right\}$ be an intuitionistic fuzzy subset of $L$. Then $A$ is called an intuitionistic fuzzy sublattice (IFL) of $L$ if the following conditions are satisfied for all $x, y \in L$ :

1. $\mu_{A}(x \vee y) \geq \min \left\{\mu_{A}(x), \mu_{A}(y)\right\}$;

2. $\mu_{A}(x \wedge y) \geq \min \left\{\mu_{A}(x), \mu_{A}(y)\right\}$;

3. $\nu_{A}(x \vee y) \leq \max \left\{\nu_{A}(x), \nu_{A}(y)\right\}$;

4. $\nu_{A}(x \wedge y) \leq \max \left\{\nu_{A}(x), \nu_{A}(y)\right\}$.

Definition 2.5. [23] An intuitionistic fuzzy set $I$ of $L$ is called an intuitionistic fuzzy ideal (IFI) of $L$, if the following conditions are satisfied for all $x, y \in L$ :

1. $\mu_{I}(x \vee y) \geq \min \left\{\mu_{I}(x), \mu_{I}(y)\right\}$

2. $\mu_{I}(x \wedge y) \geq \max \left\{\mu_{I}(x), \mu_{I}(y)\right\}$

3. $\nu_{I}(x \vee y) \leq \max \left\{\nu_{I}(x), \nu_{I}(y)\right\}$;

4. $\nu_{I}(x \wedge y) \leq \min \left\{\nu_{I}(x), \nu_{I}(y)\right\}$.

\subsection{Intuitionistic fuzzy Triangular norms and Triangular conorms}

Triangular norms and conorms (t-norms and t-conorms, for short) are important tools for the interpretation of the union and intersection of fuzzy sets [3]. Particularly, they are very useful for a lot of notions, like conjunction and disjunction in fuzzy logic, antisymmetry, and transitivity of fuzzy relations, etc.

Berthold Schweizer and Abe Sklar in [21] gave an axiomatic approach to tnorms as they are used today. Deschrijver, Cornelis, and Kerre have been extended triangular norms to intuitionistic fuzzy triangular norms $[7,8]$. In [9] Deschrijver 
and Kerre have shown that intuitionistic fuzzy sets can also be seen as $L$-fuzzy sets in the sense of Goguen [15]. Consider the set $L^{*}$ and operation defined by

$$
\begin{aligned}
L^{*} & =\left\{\left(a_{1}, a_{2}\right) \in[0,1]^{2}, a_{1}+a_{2} \leq 1\right\} \\
\left(a_{1}, a_{2}\right) & \leqslant L^{*}\left(b_{1}, b_{2}\right) \Leftrightarrow a_{1} \leq b_{1} \text { and } a_{2} \geq b_{2}, \forall\left(a_{1}, a_{2}\right),\left(b_{1}, b_{2}\right) \in L^{*}
\end{aligned}
$$

The structure $\left(L^{*}, \leqslant L^{*}\right)$ is a complete lattice [9].

We denote its units by $0_{L^{*}}=(0,1)$ and $1_{L^{*}}=(1,0)$.

Equivalently, this lattice can also be defined as an algebraic structure $\left(L^{*}, \wedge_{L^{*}}, \vee_{L^{*}}\right)$ where the meet operator $\wedge_{L^{*}}$ and the join operator $\vee_{L^{*}}$ are defined as follows, for $\left(x_{1}, x_{2}\right),\left(y_{1}, y_{2}\right) \in$ $L^{*}$ :

$$
\begin{aligned}
& \left(x_{1}, x_{2}\right) \wedge_{L^{*}}\left(y_{1}, y_{2}\right)=\left(\min \left(x_{1}, y_{1}\right), \max \left(x_{2}, y_{2}\right)\right) \\
& \left(x_{1}, x_{2}\right) \vee_{L^{*}}\left(y_{1}, y_{2}\right)=\left(\max \left(x_{1}, y_{1}\right), \min \left(x_{2}, y_{2}\right)\right)
\end{aligned}
$$

Using this lattice, we easily see that with every intuitionistic fuzzy set $A=$ $\left\{\left(x, \mu_{A}(x), \nu_{A}(x)\right) \mid x \in X\right\}$ corresponds to an $L^{*}$-fuzzy set, i.e., a mapping $A$ : $X \longrightarrow L^{*}$. In the sequel, we will use the same notation for an intuitionistic fuzzy set and its associated $L^{*}$-fuzzy set. So for the intuitionistic fuzzy set $A$ we will also use the notation

$$
A(x)=\left(\mu_{A}(x), \nu_{A}(x)\right) .
$$

In the following, we give some elementary notions and definitions on intuitionistic fuzzy sets operations, intuitionistic fuzzy t-norms and intuitionistic fuzzy t-conorms.

Definition 2.6. $[8,10]$ An intuitionistic fuzzy t-norm $\mathcal{T}$ is a commutative, associative, and increasing mapping $\mathcal{T}:\left(L^{*}\right)^{2} \longrightarrow L^{*}$ satisfying $\mathcal{T}\left(1_{L^{*}}, x\right)=x$, for all $x \in L^{*}$.

Definition 2.7. $[8,10]$ An intuitionistic fuzzy t-conorm is a commutative, associative, and increasing mapping $\mathcal{S}:\left(L^{*}\right)^{2} \longrightarrow L^{*}$ satisfying $\mathcal{S}\left(0_{L^{*}}, x\right)=x$, for all $x \in L^{*}$.

Definition 2.8. $\quad[8,10]$ An intuitionistic fuzzy t-norm $\mathcal{T}$ (resp. t-conorm $\mathcal{S}$ ) is called t-representable intuitionistic fuzzy t-norm if there exists a fuzzy t-norm $T$ and a fuzzy t-conorm $S$ (resp. a fuzzy t-conorm $S^{\prime}$ and fuzzy t-norm $T^{\prime}$ ) on $[0,1]$ such that, for all $x, y \in L^{*}, \mathcal{T}(x, y)=\left(T\left(x_{1}, y_{1}\right), S\left(x_{2}, y_{2}\right)\right)$ (resp.S $(x, y)=$ $\left(S^{\prime}\left(x_{1}, y_{1}\right), T^{\prime}\left(x_{2}, y_{2}\right) . T\right.$ and $S$ (resp. $S^{\prime}$ and $\left.T^{\prime}\right)$ are called the representants of $\mathcal{T}$ $(\operatorname{resp} \cdot \mathcal{S})$.

Also, in intuitionistic fuzzy set theory $\mathcal{S}$-union and $\mathcal{T}$-intersection can be modeled by the newly defined intuitionistic fuzzy t-norms and t-conorms. We define, for all $x \in X$ and $A, B$ intuitionistic fuzzy sets in $X$ 


$$
\begin{aligned}
& (A \cap B)(x)=\mathcal{T}(A(x), B(x)), \\
& (A \cup B)(x)=\mathcal{S}(A(x), B(x)) .
\end{aligned}
$$

Definition 2.9. [2] Let $A \in \operatorname{IFS}(X)$ where $A(x)=\left(\mu_{A}(x), \nu_{A}(x)\right)$, for $x \in X$, the sets

1. $[A](x)=\left(\mu_{A}(x), \mu_{A}^{c}(x)\right)$.

2. $\langle A\rangle(x)=\left(\nu_{A}^{c}(x), \nu_{A}(x)\right)$.

are called, respectively, necessity and possibility operators.

Definition 2.10. [2] Let $A$ be an intuitionistic fuzzy set on a set $X$. The support of $A$ is the crisp subset on $X$ given by

$$
\operatorname{Supp}(A)=\left\{x \in X \mid 0_{L^{*}}<_{L^{*}} A(x)\right\}
$$

Remark 2.1. Let $A$ be an intuitionistic fuzzy set on a set $X$. According to Definition 2.10, the support of $A$ is the crisp subset on $X$ given by $\left\{x \in X \mid \mu_{A}(x)>0\right.$ or $\mu_{A}(x)=0$ and $\left.\nu(x)<1\right\}$.

The following theorem gives the condition which makes an intuitionistic fuzzy triangular norm a representable triangular norm.

Theorem 2.1. $\quad[8,10]$ Given a fuzzy $t$-norm $T$ and a fuzzy $t$-conorm $S$ satisfying $\forall(a, b) \in[0,1], T(a, b) \leq 1-S(1-a, 1-b)$, the mappings $\mathcal{T}$ and $\mathcal{S}$ defined by, for $x, y \in L^{*}, \mathcal{T}(x, y)=\left(T\left(x_{1}, y_{1}\right), S\left(x_{2}, y_{2}\right)\right)$ and $\mathcal{S}(x, y)=\left(S\left(x_{1}, y_{1}\right), T\left(x_{2}, y_{2}\right)\right)$ are an t-representable intuitionistic fuzzy t-norm and an t-representable intuitionistic fuzzy t-conorm respectively.

Remark 2.2. If $\mathcal{T}$ (resp. $\mathcal{S}$ ) is a t-representable intuitionistic fuzzy t-norm (resp. trepresentable intuitionistic fuzzy t-conorm), we denoted by $\mathcal{T}=(T, S)(\operatorname{resp} . \mathcal{S}=(S, T))$, where

$$
\forall(a, b) \in[0,1]: S(a, b) \leq 1-T(1-a, 1-b) .
$$

Now, we give some basic examples of t-representable intuitionistic fuzzy t-norm and t-representable intuitionistic fuzzy t-conorm (see also [8]).

For all $x, y \in L^{*}$,

(i) $\mathcal{T}_{M}(x, y)=\left(\min \left(x_{1}, y_{1}\right), \max \left(x_{2}, y_{2}\right)\right)$,

(ii) $\mathcal{T}_{L}(x, y)=\left(\max \left(0, x_{1}+y_{1}-1\right), \min \left(1, x_{2}+1-y_{1}, y_{2}+1-x_{1}\right)\right)$.

Consequently, we can consider the minimum intuitionistic fuzzy t-norm and the Łukasiewicz intuitionistic fuzzy t-norm, where theire duals are 


$$
\begin{aligned}
& \mathcal{S}_{M}(x, y)=\left(\max \left(x_{1}, y_{1}\right), \min \left(x_{2}, y_{2}\right)\right) \text { and } \\
& \mathcal{S}_{L}(x, y)=\left(\min \left(1, x_{1}+1-y_{2}, y_{1}+1-x_{2}\right), \max \left(0, x_{2}+y_{2}-1\right)\right) .
\end{aligned}
$$

The following lemma will be used to prove some properties.

Lemma 2.1. Let $a \in L^{*}$. Then, $\mathcal{T}\left(a, 0_{L^{*}}\right)=0_{L^{*}}$.

Proof. Let $a \in L^{*}$. We have $\mathcal{T}\left(a, 0_{L^{*}}\right) \leqslant L_{L^{*}} \mathcal{T}\left(1_{L^{*}}, 0_{L^{*}}\right)=0_{L^{*}}$. Then, $\mathcal{T}\left(a, 0_{L^{*}}\right)=$ $0_{L^{*}}$.

Conclusion 2.1. Consider the lattice $L^{*}$ and $0_{L^{*}} \in L^{*}$, then $\mathcal{T}\left(0_{L^{*}}, 0_{L^{*}}\right)=0_{L^{*}}$.

Proposition 2.1. Let $a, b \in L^{*}$. Then,

1. If $0_{L^{*}}<_{L^{*}}$ a and $0_{L^{*}}<_{L^{*}} b$ then $0_{L^{*}}<_{L^{*}} \mathcal{T}(a, b)$,

2. $0_{L^{*}}<_{L^{*}} \mathcal{T}_{M}(a, b)$ then $\left(0_{L^{*}}<_{L^{*}}\right.$ a and $\left.0_{L^{*}}<_{L^{*}} b\right)$,

3. $\mathcal{T}_{M}(a, a)=a$,

4. $a \leqslant L^{*} \mathcal{S}(a, b)$,

5. $\mathcal{T}(a, b) \leqslant L^{*} a$,

6. $a=\mathcal{T}_{M}(a, b)$ if and anly if $a \leqslant L^{*} b$.

Proof. Let $a, b \in L^{*}$.

1. Using the property of $\mathcal{T}$ is monotonicity and Conclusion 2.1.

2. Assume that $0_{L^{*}}=a$ or $0_{L^{*}}=b$, then, $\mathcal{T}_{M}(a, b)=0_{L^{*}}$.

3. Using the definition of $\mathcal{T}_{M}=\wedge_{M}$.

4. We have $a=\mathcal{S}\left(a, 0_{L^{*}}\right) \leqslant_{L^{*}} \mathcal{S}(a, b)$. Then, $a \leqslant L_{L^{*}} \mathcal{S}(a, b)$.

5. We have $\mathcal{T}(a, b) \leqslant_{L^{*}} \mathcal{T}\left(a, 1_{L^{*}}\right)=a$. Then, $\mathcal{T}(a, b) \leqslant_{L^{*}} a$.

6. Using the property of $\mathcal{T}_{M}$.

Throughout this paper, all intuitionistic fuzzy t-norms $\mathcal{T}$ ( respectively intuitionistic fuzzy t-conorms $\mathcal{S}$ ) are t-representable intuitionistic fuzzy t-norm (resp, t-representable intuitionistic fuzzy t-conorm).

The following definition inspired from $[18,19]$ extends the notion dominance to the intuitionistic fuzzy case. 
Definition 2.11. Let $\mathcal{T}_{1}$ and $\mathcal{T}_{2}$ be two t-representable intuitionistic fuzzy tnorms. $\mathcal{T}_{1}$ is said to dominate another t-norm $\mathcal{T}_{2}$ (briefly, $\mathcal{T}_{2} \ll \mathcal{T}_{1}$ ) if and only if, for any quadruple $\left(x=\left(x_{1}, x_{2}\right), y=\left(y_{1}, y_{2}\right), u=\left(u_{1}, u_{2}\right), v=\left(v_{1}, v_{2}\right)\right) \in\left(L^{*}\right)$, the following holds:

$$
\mathcal{T}_{2}\left(\mathcal{T}_{1}(x, u), \mathcal{T}_{1}(y, v)\right) \leqslant L^{*} \mathcal{T}_{1}\left(\mathcal{T}_{2}(x, y), \mathcal{T}_{2}(u, v)\right)
$$

The following lemma will be used to prove the subsequent results.

Lemma 2.2. For any be a t-representable intuitionistic fuzzy $t$-norm $\mathcal{T}$, we have $\mathcal{T} \ll$ $\mathcal{T}_{M}$

Proof. Let $x, y, u, v \in L^{*}$,

$$
\left\{\begin{array}{l}
\mathcal{T}_{M}(x, y) \leqslant L^{*} x \text { and } \mathcal{T}_{M}(u, v) \leqslant L_{L^{*}} u \\
\mathcal{T}_{M}(x, y) \leqslant L^{*} y \text { and } \mathcal{T}_{M}(u, v) \leqslant L^{*} v
\end{array}\right.
$$

Then,

$$
\left\{\begin{array}{l}
\mathcal{T}\left(\mathcal{T}_{M}(x, y), \mathcal{T}_{M}(u, v)\right) \leqslant L_{L^{*}} \mathcal{T}(x, u) \\
\mathcal{T}\left(\mathcal{T}_{M}(x, y), \mathcal{T}_{M}(u, v)\right) \leqslant L^{*} \mathcal{T}(y, v)
\end{array}\right.
$$

Hence,

$$
\begin{aligned}
& \mathcal{T}_{M}\left(\mathcal{T}\left(\mathcal{T}_{M}(x, y), \mathcal{T}_{M}(u, v)\right), \mathcal{T}\left(\mathcal{T}_{M}(x, y), \mathcal{T}_{M}(u, v)\right)\right) \leqslant L^{*} \\
& \mathcal{T}_{M}(\mathcal{T}(x, u), \mathcal{T}(y, v)) .
\end{aligned}
$$

So,

$$
\mathcal{T}\left(\mathcal{T}_{M}(x, y), \mathcal{T}_{M}(u, v)\right) \leqslant L^{*} \mathcal{T}_{M}(\mathcal{T}(x, u), \mathcal{T}(y, v))
$$

Lemma 2.3. Any t-representable intuitionistic fuzzy t-norm $\mathcal{T}$ dominates itself, i.e., for any quadruple $(x, y, u, v) \in\left(L^{*}\right)^{4}$, we have

$$
\mathcal{T}(\mathcal{T}(x, u), \mathcal{T}(y, v))=\mathcal{T}(\mathcal{T}(x, y), \mathcal{T}(u, v)) .
$$

Proof. Direct.

Remark 2.3. $\quad$ 1. The greatest t-representable intuitionistic fuzzy t-norm w.r.t $\leqslant_{L^{*}}$ is $\mathcal{T}_{M}$, defined by $\mathcal{T}_{M}(x, y)=x \wedge_{L^{*}} y$.

2. The smallest t-representable intuitionistic fuzzy t-conorm w. r. $\mathrm{t} \leqslant L_{L^{*}}$ is $\mathcal{S}_{M}$, defined by $\mathcal{S}_{M}(x, y)=x \vee_{L^{*}} y$ for all $x, y \in L^{*}$.

Moreover, $\mathcal{T}_{M}(x, y) \leqslant L^{*} \mathcal{S}_{M}(x, y)$ for all $x, y \in L^{*}$. Indeed, let $x, y \in L^{*}$, where $x=\left(x_{1}, x_{2}\right)$ and $y=\left(y_{1}, y_{2}\right)$.

We have $\min \left(x_{1}, y_{1}\right) \leq \max \left(x_{1}, y_{1}\right)$ and $\max \left(x_{2}, y_{2}\right) \geq \min \left(x_{2}, y_{2}\right)$,

then $\left(\min \left(x_{1}, y_{1}\right), \max \left(x_{2}, y_{2}\right)\right) \leqslant_{L^{*}}\left(\max \left(x_{1}, y_{1}\right), \min \left(x_{2}, y_{2}\right)\right)$,

then $\mathcal{T}_{M}(x, y) \leqslant L^{*} \mathcal{S}_{M}(x, y)$. 
Remark 2.4. It easy to see that $\mathcal{T}(x, y) \leqslant_{L^{*}} \mathcal{T}_{M}(x, y)$ and $\mathcal{S}_{M}(x, y) \leqslant_{L^{*}} \mathcal{S}(x, y)$ for all $x, y \in L^{*}$. Indeed, let $x, y \in L^{*}$, then $x=\left(x_{1}, x_{2}\right)$ and $y=\left(y_{1}, y_{2}\right)$. We have $T\left(x_{1}, y_{1}\right) \leq$ $\min \left(x_{1}, y_{1}\right)$ and $S\left(x_{2}, y_{2}\right) \geq \max \left(x_{2}, y_{2}\right)$ then $\mathcal{T}(x, y) \leqslant_{L^{*}} \mathcal{T}_{M}(x, y)$ and $\mathcal{S}_{M}(x, y) \leqslant_{L^{*}}$ $\mathcal{S}(x, y)$.

Finaly, $\mathcal{T}(x, y) \leqslant_{L^{*}} \mathcal{T}_{M}(x, y) \leqslant L^{*} \mathcal{S}_{M}(x, y) \leqslant L^{*} \mathcal{S}(x, y)$

Remark 2.5. Note that it does not hold that for all $x, y \in L^{*}$, neither $\mathcal{T}_{M}(x, y)=x$ nor $\mathcal{T}_{M}(x, y)=y$. For example, $\mathcal{T}_{M}((0.5,0.3),(0.2,0.1))=(0.2,0.3)$.

\section{3. $\quad \mathcal{T}$-Intuitionistic fuzzy sublattices}

In this section, we introduce and study the notion of an intuitionistic fuzzy sublattice w. r. $t$ a t-representable intuitionistic fuzzy t-norm, intuitionistic fuzzy ideal w. r. $t$ a t-representable intuitionistic fuzzy t-norm. Also, their characterizations w. r. t a t-representable intuitionistic fuzzy t-norm.

Definition 3.1. Let $\mathcal{T}$ be a t-representable intuitionistic fuzzy t-norm, $L$ a crisp lattice and $A$ an intuitionistic fuzzy set. $A$ is called an intuitionistic fuzzy sublattice on lattice $L$ w. r. t the t-representable intuitionistic fuzzy t-norm $\mathcal{T}$ if and only if the following inequality holds

$$
\mathcal{T}(A(x), A(y)) \leqslant_{L^{*}} A(x \vee y) \wedge_{L^{*}} A(x \wedge y)
$$

Notation 3.1. The set of all the intuitionistic fuzzy lattices on a lattice $L w . r . t$ a t-representable intuitionistic fuzzy $t$-norm $\mathcal{T}$ will be denoted by $\mathcal{T}$-IFL $(L)$.

Remark 3.1. If $A \in \mathcal{T}_{M}-I F L(L)$, then $A$ is an intuitionistic fuzzy lattice on lattice $L$, within the meaning of K. V. Thomas and L. S. Nair in [23]. So intuitionistic fuzzy lattice w. $r$. $\mathrm{t}$ the t-representable intuitionistic fuzzy t-norm $\mathcal{T}$ is a generalization of the intuitionistic fuzzy lattice.

Example 3.1. Consider the lattice $L=\{1,2,3,6\}$ the set of all divisors of 6 . Let $A$ be the intuitionistic fuzzy set given by $A(1)=(0.1,0), A(2)=(0.4,0.3), A(3)=$ $(0.5,0.5), A(6)=(0.2,0.3)$.

Then, $A$ is a $\mathcal{T}_{L}-I F L(L)$. Indeed, it suffices to prove it for $x, y \in L$ we get that 


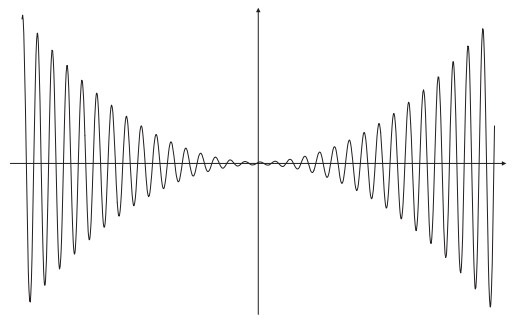

Fig. 3.1: The Hasse diagram of $D(6)$

\begin{tabular}{|c|c|c|c|c|c|c|c|}
\hline$x$ & $y$ & $A(x)$ & $A(y)$ & $x \wedge y$ & $A(x \wedge y)$ & $x \vee y$ & $A(x \vee y)$ \\
\hline 1 & 1 & $(0.1,0.2)$ & $(0.1,0.2)$ & 1 & $(0.1,0.2)$ & 1 & $(0.1,0.2)$ \\
\hline 1 & 2 & $(0.1,0.2)$ & $(0.4,0.3)$ & 1 & $(0.1,0.2)$ & 2 & $(0.4,0.3)$ \\
\hline 1 & 3 & $(0.1,0.2)$ & $(0.5,0.5)$ & 1 & $(0.1,0.2)$ & 3 & $(0.5,0.5)$ \\
\hline 1 & 6 & $(0.1,0.2)$ & $(0.2,0.3)$ & 1 & $(0.1,0.2)$ & 6 & $(0.2,0.3)$ \\
\hline 2 & 1 & $(0.4,0.3)$ & $(0.1,0.2)$ & 1 & $(0.1,0.2)$ & 2 & $(0.4,0.3)$ \\
\hline 2 & 2 & $(0.4,0.3)$ & $(0.4,0.3)$ & 2 & $(0.4,0.3)$ & 2 & $(0.4,0.3)$ \\
\hline 2 & 3 & $(0.4,0.3)$ & $(0.5,0.5)$ & 1 & $(0.1,0.2)$ & 6 & $(0.2,0.3)$ \\
\hline 2 & 6 & $(0.4,0.3)$ & $(0.2,0.3)$ & 2 & $(0.4,0.3)$ & 6 & $(0.2,0.3)$ \\
\hline 3 & 1 & $(0.5,0.5)$ & $(0.1,0.2)$ & 1 & $(0.1,0.2)$ & 3 & $(0.5,0.5)$ \\
\hline 3 & 2 & $(0.5,0.5)$ & $(0.4,0.3)$ & 1 & $(0.1,0.2)$ & 6 & $(0.4,0.3)$ \\
\hline 3 & 3 & $(0.5,0.5)$ & $(0.5,0.5)$ & 3 & $(0.5,0.5)$ & 3 & $(0.5,0.5)$ \\
\hline 3 & 6 & $(0.5,0.5)$ & $(0.2,0.3)$ & 3 & $(0.5,0.5)$ & 6 & $(0.2,0.3)$ \\
\hline 6 & 1 & $(0.2,0.3)$ & $(0.1,0.2)$ & 1 & $(0.1,0.2)$ & 6 & $(0.2,0.3)$ \\
\hline 6 & 2 & $(0.2,0.3)$ & $(0.4,0.3)$ & 2 & $(0.4,0.3)$ & 6 & $(0.2,0.3)$ \\
\hline 6 & 3 & $(0.2,0.3)$ & $(0.5,0.5)$ & 3 & $(0.5,0.5)$ & 6 & $(0.2,0.3)$ \\
\hline 6 & 6 & $(0.2,0.3)$ & $(0.2,0.3)$ & 6 & $(0.2,0.3)$ & 6 & $(0.2,0.3)$ \\
\hline
\end{tabular}




\begin{tabular}{|c|c|c|}
\hline $\mathcal{T}_{L}(A(x),(A(y))$ & $A(x \wedge y) \wedge_{L^{*}} A(x \vee y)$ & The inequality 3.1 \\
\hline$(0,0.4)$ & $(0.1,0.2)$ & True \\
\hline$(0,0.5)$ & $(0.1,0.3)$ & True \\
\hline$(0,0.7)$ & $(0.1,0.5)$ & True \\
\hline$(0,0.5)$ & $(0.1,0.3)$ & True \\
\hline$(0,0.5)$ & $(0.1,0.3)$ & True \\
\hline$(0,0.6)$ & $(0.4,0.3)$ & True \\
\hline$(0,0.8)$ & $(0.1,0.3)$ & True \\
\hline$(0,0.6)$ & $(0.2,0.3)$ & True \\
\hline$(0,0.7)$ & $(0.1,0.5)$ & True \\
\hline$(0,0.8)$ & $(0.5,0.5)$ & True \\
\hline$(0,1)$ & $(0.2,0.5)$ & True \\
\hline$(0,0.8)$ & $(0.1,0.3)$ & True \\
\hline$(0,0.5)$ & $(0.2,0.3)$ & True \\
\hline$(0,0.6)$ & $(0.2,0.5)$ & True \\
\hline$(0,0.8)$ & $(0.2,0.3)$ & True \\
\hline$(0,0.6)$ & $(0.1,0.5)$ & True \\
\hline
\end{tabular}

The following lemma is immediate and shows the generality of our work.

Lemma 3.1. If $A \in \mathcal{T}_{M}-I F L(L)$ then $A \in \mathcal{T}-I F L(L)$, the converse is note true.

Proof. Assume that $A \in \mathcal{T}_{M^{-}} I F L(L)$. Then $\mathcal{T}_{M}(A(x), A(y)) \leqslant_{L^{*}} A(x \vee y) \wedge_{L^{*}}$ $A(x \wedge y)$. Using the Remark 2.4, then $\mathcal{T}(A(x), A(y)) \leqslant L_{L^{*}} A(x \vee y) \wedge L_{L^{*}} A(x \wedge y)$. So $A \in \mathcal{T}-\operatorname{IFL}(L)$. The converse in general is not true. Indeed, let $L$ be the lattice in Example 3.1 and let $A$ given by $A(1)=(0.1,0.2), A(2)=(0.4,0.3), A(3)=$ $(0.5,0.5), A(6)=(0.2,0.3)$. Then $A$ is an $\mathcal{T}_{L^{-}} I F L(L)$ but $A$ is note an $\mathcal{T}_{M^{-}}$ IFL $(L)$. But $\mathcal{T}_{M}\left(A(2),(A(3))=(0.4,0.5) \nless_{L^{*}}(0.1,0.3)=A(2 \wedge 3) \wedge_{L^{*}} A(2 \vee 3)=\right.$ $A(1) \wedge_{L^{*}} A(6)$. Then $A$ is note an $\mathcal{T}_{M}-I F L(L)$.

The following theorems about the intersection of two $\mathcal{T}$-intuitionistic fuzzy lattices on lattice $L$

Theorem 3.2. The $\mathcal{T}$-intersection of two $\mathcal{T}$-intuitionistic fuzzy sublattices on a lattice $L$ is a $\mathcal{T}$-intuitionistic fuzzy sublattice on the lattice $L$.

Proof. Let $A, B \in \mathcal{T}$ - $I F L(L)$, i.e., $\mathcal{T}(A(x), A(y)) \leqslant_{L^{*}} A(x \wedge y) \wedge_{L^{*}} A(x \vee y)$ and $\mathcal{T}(B(x), B(y)) \leqslant_{L^{*}} B(x \wedge y) \wedge_{L^{*}} B(x \vee y)$. We have for $x \in L:(A \cap B)(x)=$ $\mathcal{T}(A(x), B(x))$. Using the Lemma 2.2 and 2.3 .

We have $\mathcal{T}(\mathcal{T}(A(x), A(y)), \mathcal{T}(B(x), B(y))) \leqslant L^{*}$

$\mathcal{T}\left(A(x \wedge y) \wedge_{L^{*}} A(x \vee y), B(x \wedge y) \wedge_{L^{*}} B(x \vee y)\right)$

Hence, $\mathcal{T}(\mathcal{T}(A(x), B(x)), \mathcal{T}(A(y), B(y))) \leqslant L^{*}$ $\mathcal{T}(A(x \wedge y), B(x \wedge y)) \wedge_{L^{*}} \mathcal{T}(A(x \vee y), B(x \vee y))$

Thus, $\mathcal{T}((A \cap B)(x),(A \cap B)(y)) \leqslant_{L^{*}}$ $(A \cap B)(x \wedge y) \wedge_{L^{*}}(A \cap B)(x \vee y)$. Then, $A \cap B \in \mathcal{T}-I F L(L)$. 
Corollary 3.1. Let $L$ be a lattice. If $A \in \mathcal{T}-\operatorname{IFL}(L)$ and $B \in \mathcal{T}_{M}-\operatorname{IFL}(L)$, then $A \cap B \in \mathcal{T}-I F L(L)$.

Proof. Obviously according to Lemma 3.1.

The following theorem characterizes the intersection of two $\mathcal{T}$-intuitionistic fuzzy sublattices on lattice $L$, where the intersection of intuitionistic fuzzy sets is given w. r. t another t-representable intuitionistic fuzzy t-norm $\mathcal{T}$.

Theorem 3.3. Let $L$ be a lattice, $A, B \in \mathcal{T}$-IFL $(L)$ and let $\mathcal{T}^{\prime}$ be a $t$-representable intuitionistic fuzzy t-norm dominates $\mathcal{T}$. Then, the $\mathcal{T}^{\prime}$-intersection of $A$ and $B$ is a $\mathcal{T}^{\prime}-I F L(L)$.

Proof. Suppose that $\mathcal{T}$ is dominated by $\mathcal{T}^{\prime}$.

Let $A$ and $B$ be in $\mathcal{T}$ - $I F L(L)$ i.e.,

$\mathcal{T}(A(x), A(y)) \leqslant_{L^{*}} A(x \wedge y) \wedge_{L^{*}} A(x \vee y)$ and

$\mathcal{T}(B(x), B(y)) \leqslant L^{*} B(x \wedge y) \wedge L_{L^{*}} B(x \vee y)$ for any $x, y \in L$. Then

$\mathcal{T}^{\prime}(\mathcal{T}(A(x), A(y)), \mathcal{T}(B(x), B(y)))$

$\leqslant_{L^{*}} \mathcal{T}^{\prime}\left(A(x \wedge y) \wedge_{L^{*}} A(x \vee y), B(x \wedge y) \wedge_{L^{*}} B(x \vee y)\right)$

From $\mathcal{T} \ll \mathcal{T}^{\prime}$, Lemma 2.2 and the transitivity of $\leqslant L^{*}$ it follows that

$\mathcal{T}\left(\mathcal{T}^{\prime}(A(x), B(x)), \mathcal{T}^{\prime}(A(y), B(y))\right)$

$\leqslant \mathcal{T}^{\prime}(A(x \wedge y), B(x \wedge y)) \wedge_{L^{*}} \mathcal{T}^{\prime}(A(x \vee y), B(x \vee y))$

As $(A \cap B)(x)=\mathcal{T}^{\prime}(A(x), B(x))$ for any $x \in L$, then, $\mathcal{T}((A \cap B)(x),(A \cap B)(y)) \leqslant_{L^{*}}(A \cap B)(x \wedge y) \wedge L_{L^{*}}(A \cap B)(x \vee y)$.

So, $A \cap B \in \mathcal{T}^{\prime}-I F L(L)$.

Remark 3.2. The union of two $\mathcal{T}-I F L s$ need not be a $\mathcal{T}-I F L$. Indeed, consider the lattice given in Example 3.1 and define $A, B$ by $A(1)=(0.7,0.2), A(2)=(0.4,0.5), A(3)=$ $(0.1,0.5), A(6)=(0.2,0.4)$ and $B(1)=(0.6,0.1), B(2)=(0.1,0.5), B(3)=(0.3,0.3), B(6)=$ $(0.2,0.3)$.

It is easy to see that $A$ and $B$ are $\mathcal{T}$-IFLs of $L$. If we put $(A \cup B)(x)=\mathcal{S}_{M}(A(x), B(x))$ for all $x \in L .(A \cup B)(1)=(0.7,0.1),(A \cup B)(2)=(0.4,0.5),(A \cup B)(3)=(0.3,0.3),(A \cup B)(6)=$ $(0.2,0.3)$.

But, $\mathcal{T}_{M}((A \cup B)(3),(A \cup B)(2))=(0.3,0.5) \nless_{L^{*}}$

$\mathcal{T}((A \cup B)(3 \wedge 2),(A \cup B)(3 \vee 2))=\mathcal{T}((A \cup B)(1),(A \cup B)(6))$

$=(0.2,0.3)$.

So $A \cup B$ is not a $\mathcal{T}-I F L$.

Proposition 3.1. Let $L$ be a lattice. For any t-representable intuitionistic fuzzy t-norm $\mathcal{T}$, if $A$ is a $\mathcal{T}$-IFL $(L)$ then $[A]$ and $\langle A\rangle$ are $\mathcal{T}$-IFLs of $L$.

Proof. Let $L$ be a lattice and assume that $A$ is a $\mathcal{T}-I F L(L)$. We have $[A]=$ $\left\{\langle x,[A](x)\rangle \mid[A](x) \in L^{*}\right\}$, where 


$$
\begin{aligned}
& {[A](x)=\left(\mu_{A}(x), \mu_{A}^{c}(x)\right) \text { and } \mu_{A}(x)+\mu_{A}^{c}(x)=1 . \text { Then for any } x, y \in L,} \\
& A \in \mathcal{T}-I F L(L) \text { this implies } \mathcal{T}(A(x), A(y)) \leqslant L^{*} A(x \vee y) \wedge_{L^{*}} A(x \wedge y) \\
& \Rightarrow\left(T\left(\mu_{A}(x), \mu_{A}(y)\right), S\left(\nu_{A}(x), \nu_{A}(y)\right)\right) \leqslant L^{*} \\
& \left(\min \left(\mu_{A}(x \vee y), \mu_{A}(x \wedge y)\right), \max \left(\nu_{A}(x \vee y), \nu_{A}(x \wedge y)\right)\right) \\
& \Rightarrow\left\{\begin{array}{c}
T\left(\mu_{A}(x), \mu_{A}(y)\right) \leq \min \left(\mu_{A}(x \vee y), \mu_{A}(x \wedge y)\right) \\
S\left(\nu_{A}(x), \nu_{A}(y)\right) \geq \max \left(\nu_{A}(x \vee y), \nu_{A}(x \wedge y)\right)
\end{array}\right. \\
& \Rightarrow T\left(\mu_{A}(x), \mu_{A}(y)\right) \leq \min \left(\mu_{A}(x \vee y), \mu_{A}(x \wedge y)\right) \\
& \Rightarrow 1-T\left(\mu_{A}(x), \mu_{A}(y)\right) \geq 1-\min \left(\mu_{A}(x \vee y), \mu_{A}(x \wedge y)\right) \\
& \Rightarrow S\left(1-\mu_{A}(x), 1-\mu_{A}(y)\right) \geq \max \left(1-\mu_{A}(x \vee y), 1-\mu_{A}(x \wedge y)\right) \\
& \Rightarrow S\left(\mu_{A}^{c}(x), \mu_{A}^{c}(y)\right) \geq \max \left(\mu_{A}^{c}(x \vee y), \mu_{A}^{c}(x \wedge y)\right) .
\end{aligned}
$$

Then

$$
\begin{aligned}
\mathcal{T}([A](x),[A](y))= & \mathcal{T}\left(\left(\mu_{A}(x), \mu_{A}^{c}(x)\right),\left(\mu_{A}(y), \mu_{A}^{c}(y)\right)\right), \\
= & \left(T\left(\mu_{A}(x), \mu_{A}(y)\right), S\left(\mu_{A}^{c}(x), \mu_{A}^{c}(y)\right)\right), \\
\leqslant & \left(\begin{array}{c}
\min \left(\mu_{A}(x \vee y), \mu_{A}(x \wedge y)\right) \\
, \max \left(\mu_{A}^{c}(x \vee y), \mu_{A}^{c}(x \wedge y)\right)
\end{array}\right), \\
= & \left(\mu_{A}(x \vee y), \mu_{A}^{c}(x \vee y)\right) \\
& \wedge_{L^{*}}\left(\mu_{A}(x \wedge y), \mu_{A}^{c}(x \wedge y)\right),
\end{aligned}
$$

Hence $[A]$ is a $\mathcal{T}-I F L$ of $L$.

Concerning the set $\langle A\rangle$, we have for any $x, y \in L$,

$$
\begin{array}{cl} 
& \mathcal{T}(\langle A\rangle(x),\langle A\rangle(y))=\mathcal{T}\left(\left(\nu_{A}^{c}(x), \nu_{A}(x)\right),\left(\nu_{A}^{c}(y), \nu_{A}(y)\right)\right), \\
=\quad & \left(T\left(\nu_{A}^{c}(x), \nu_{A}^{c}(y)\right), S\left(\nu_{A}(x), \nu_{A}(y)\right)\right), \\
\leqslant_{L^{*}} & \left(\min \left(\nu_{A}^{c}(x \vee y), \nu_{A}^{c}(x \wedge y)\right), \max \left(\nu_{A}(x \vee y), \nu_{A}(x \wedge y)\right)\right), \\
=\quad & \left(\left(\nu_{A}^{c}(x \vee y), \nu_{A}(x \vee y)\right) \wedge_{L^{*}}\left(\nu_{A}^{c}(x \wedge y), \nu_{A}(x \wedge y)\right)\right), \\
=\quad & \langle A\rangle(x \vee y) \wedge_{L^{*}}\langle A\rangle(x \wedge y) .
\end{array}
$$

Hence $\langle A\rangle$ is a $\mathcal{T}-I F L$ of $L$.

Proposition 3.2. Let $L$ be a lattice. For any t-representable intuitionistic fuzzy t-norm $\mathcal{T}$, if $A$ is a $\mathcal{T}$-IFL $(L)$ then supp $(A)$ is a crisp sublattice of $L$.

Proof. Let $x, y \in \operatorname{supp}(A)$. Using Propriety 2.1. Then $0_{L^{*}}<_{L^{*}} A(x)$ and $0_{L^{*}}<_{L^{*}}$ $A(y)$. Since $\mathcal{T}(A(x), A(y)) \leqslant_{L^{*}} A(x \vee y) \wedge_{L^{*}} A(x \wedge y)$ it follows that $0_{L^{*}}<L_{L^{*}}$ $A(x \vee y) \wedge \wedge_{L^{*}} A(x \wedge y)$. So $0_{L^{*}}<_{L^{*}} A(x \vee y)$ and $0_{L^{*}}<L_{L^{*}} A(x \wedge y)$. Hence $x \vee y \in$ $\operatorname{supp}(A)$ and $x \wedge y \in \operatorname{supp}(A)$. Thus $\operatorname{supp}(A)$ is a crisp sublattice of $L$.

Remark 3.3. The converse of the above Proposition 3.2 does not holds in general. Indeed, let $L$ be the lattice in Example 3.1 and $A \in I F L(L)$ given by $A(1)=(0.4,0.3), A(2)=$ $(0.7,0.2), A(3)=(0.2,0.2), A(6)=(0.4,0.1)$. Obviously $\operatorname{supp}(A)=\{1,2,3,6\}=L$ is a crisp lattice. Since $\mathcal{T}_{M}(A(2), A(3))=(0.2,0.2)$ and $\Varangle_{L^{*}} A(2 \vee 3) \wedge_{L^{*}} A(2 \wedge 3)=$ $A(6) \wedge_{L^{*}} A(1)=(0.4,0.3)$, it follows that $A$ is not an intuitionistic fuzzy lattice of $L$. 


\section{4. $\mathcal{T}$-Intuitionistic fuzzy ideal}

The notion of an intuitionistic fuzzy ideal on a lattice was first introduced by Thomas and Nair [23]. In this section, we give a new characterization of intuitionistic fuzzy ideals on a lattice w. r. t a given intuitionistic fuzzy t-norm.

Definition 4.1. Let $L$ be a lattice, $\mathcal{T}$ be a t-representable intuitionistic fuzzy t-norm, $\mathcal{S}$ the intuitionistic triangular conorm associated to $\mathcal{T}$ and let $I$ be an intuitionistic fuzzy set. $I$ is called an intuitionistic fuzzy ideal on the lattice $L$ w.r.t $\mathcal{T}$ if the following conditions are satisfied:

1. $\mathcal{T}(I(x), I(y)) \leqslant L^{*} I(x \vee y)$,

2. $\mathcal{S}(I(x), I(y)) \leqslant L^{*} I(x \wedge y)$.

Notation 4.1. The set of all the intuitionistic fuzzy ideals on lattice $L w . r$. $t$ an intuitionistic fuzzy t-norm $\mathcal{T}$ will be denoted by $\mathcal{T}$-IFI $(L)$.

Example 4.1. Consider the lattice $L$ of "all integer divisors of 18 ". That is $L=$ $\{1,2,3,6,9,18\}$. Let $A$ be an intuitionistic fuzzy set given by $A(1)=(0.7,0.2), A(2)=$ $(0.5,0.5), A(3)=(0.6,0.3), A(6)=(0.4,0.5), A(9)=(0.5,0.5), A(18)=(0.4,0.5)$. It easy to see that $A$ is a $\mathcal{T}-I F I(L)$.

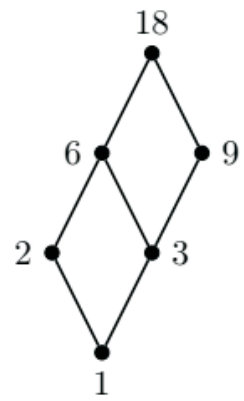

FIG. 4.1: The Hasse diagram of $D(18)$

Remark 4.1. In general, a $\mathcal{T}_{M}-I F I(L)$ is not a $\mathcal{T}-I F I(L)$. Indeed, let $L$ be the lattice in Example 3.1 and $A \in I F S(L)$ given by $A(1)=(0.4,0.2), A(2)=(0.4,0.3), A(3)=$ $(0.1,0.3), A(6)=(0.1,0.3)$. Obviously $A \in \mathcal{T}_{M}-I F I(L)$. Since $\mathcal{S}_{L}(A(2), A(3))=$ $(0.8,0) \nless_{L^{*}} A(2 \wedge 3)=A(1)=(0.4,0.2)$, it follows that $A$ is not $\mathcal{T}_{L}-I F I(L)$.

Remark 4.2. Every $\mathcal{T}_{M^{-}} I F I(L)$ is a $\mathcal{T}_{M}-I F L(L)$. Indeed, if $I$ is $\mathcal{T}_{M}-I F I(L)$, then $\mathcal{T}_{M}(I(x), I(y)) \leqslant_{L^{*}} I\left((x \vee y)\right.$ and $\mathcal{S}_{M}(I(x), I(y)) \leqslant_{L^{*}} I((x \wedge y)$, according with Remark 2.3 , it is not difficult to see that $\mathcal{T}_{M}(I(x), I(y)) \leqslant_{L^{*}} I(x \vee y) \wedge_{L^{*}} I(x \wedge y)$, then $I$ 
is a $\mathcal{T}_{M}-I F L(L)$. The converse is not true as seen in the following example. Consider the lattice $L$ given in Example 3.1. Define $A$ to be $A(1)=(0.3,0.2), A(2)=(0.2,0.4), A(3)=$ $(0.2,0.6), A(6)=(0.5,0.4)$. Hence $A$ is a $\mathcal{T}_{M}-I F L(L)$, but not a $\mathcal{T}_{M}-I F I(L)$, because $\mathcal{S}_{M}(I(3), I(6))=(0.5,0.4) \nless_{L^{*}} I(3 \wedge 6)=I(3)=(0.2,0.6)$.

Lemma 4.1. If $I \in \mathcal{T}-I F I(L)$ then $I \in \mathcal{T}_{M}-I F L(L)$.

Proof. Let $L$ be a lattice and let $I$ is $\mathcal{T}-I F I(L)$ then $\mathcal{T}(I(x), I(y)) \leqslant_{L^{*}} I(x \vee y)$ and $\mathcal{S}(I(x), I(y)) \leqslant L^{*} I(x \wedge y)$. According to Lemma 2.4 and Propriety 2.1 we obtain the result.

Remark 4.3. The converse of the Lemma 4.1 does not hold in general. Indeed, let $L$ be the lattice of "all integers divisors of 6 " and $A$ an $\operatorname{IFS}(L)$ defined by $A(1)=$ $(0.1,0), A(2)=(0.4,0.3), A(3)=(0.1,0.2), A(6)=(0.1,0.3)$. Trivially, that $A$ is a $\mathcal{T}_{M^{-}}$ $I F L(L)$. But, since $\mathcal{S}_{M}(A(1), A(2))=\mathcal{S}_{M}((0.1,0),(0.4,0.3))=(0.4,0) \Varangle_{L^{*}} A(1 \vee 2)=$ $A(6)=(0.1,0.3)$. It holds that $A$ is not a $\mathcal{T}-I F I(L)$

Theorem 4.2. For any $I \in \mathcal{T}$-IFI $(L)$, then $I \in \mathcal{T}$-IFL $(L)$.

Proof. Using Lemma 4.1 and Lemma 3.1 the theorem holds.

Conclusion 4.1. Every $\mathcal{T}_{M}-I F I(L)$ is a $\mathcal{T}-I F L(L)$.

In the following, we start with the key results.

As a corollary, we obtain the following interesting theorem of $\mathcal{T}$-IFI $(L)$.

Lemma 4.2. Let $L$ be a lattice and $I \in \mathcal{T}$-IFI $(L)$. Then for any $x, y \in L$, if $x \leq y$, then $I(y) \leqslant L^{*} I(x)$.

Proof. Direct from [20, Corollary 3.1].

In the following theorem, we provide a basic characterization of $\mathcal{T}_{M}$-IFI $(L)$.

Theorem 4.3. Let $L$ be a lattice. Then it holds that $I$ is a $\mathcal{T}_{M}-I F I(L)$ if and only if the following condition is satisfied:

$$
I(x \vee y)=\mathcal{T}_{M}(I(x), I(y)) .
$$

Proof. Suppose that $I$ is a $\mathcal{T}_{M^{-}} I F I(L)$. Then $\mathcal{T}_{M}(I(x), I(y)) \leqslant L^{*} I(x \vee y)$. Since $\left\{\begin{array}{l}x \leq x \vee y, \\ y \leq x \vee y .\end{array}\right.$ we obtain from Lemma 4.2 that $\left\{\begin{array}{l}I(x \vee y) \leqslant L^{*} I(x), \\ I(x \vee y) \leqslant L^{*} I(y) .\end{array}\right.$

Then, $\mathcal{T}_{M}(I(x \vee y), I(x \vee y)) \leqslant L_{L^{*}} \mathcal{T}_{M}(I(x), I(y))$. Hence $I(x \vee y) \leqslant_{L^{*}} \mathcal{T}_{M}(I(x), I(y))$. So $I(x \vee y)=\mathcal{T}_{M}(I(x), I(y))$.

Conversely, suppose that $I(x \vee y)=\mathcal{T}_{M}(I(x), I(y))$, for any $x, y \in L$. Then it is easy to see that $\mathcal{T}_{M}(I(x), I(y)) \leqslant L^{*} I(x \vee y)$, for any $x, y \in L^{*}$. Next, 
we will show that $\mathcal{S}_{M}(I(x), I(y)) \leqslant L^{*} I(x \wedge y)$, for any $x, y \in L$. Let $x, y \in L$, since $x \vee(x \wedge y)=x$ and $y \vee(x \wedge y)=y$. Then it holds that $I(x \vee(x \wedge y))=$ $I(x)$ and $I(y \vee(x \wedge y))=I(y)$. From hypothesis (4.1) is it follows that $I(x)=$ $\mathcal{T}_{M}(I(x), I(x \wedge y))$ and $I(y)=\mathcal{T}_{M}(I(y), I(x \wedge y))$. Hence, $I(x) \leqslant L_{L^{*}} I(x \wedge y)$ and $I(y) \leqslant_{L^{*}} I(x \wedge y)$. Thus, $\mathcal{S}_{M}(I(x), I(y)) \leqslant_{L^{*}} I(x \wedge y)$, for any $x, y \in L^{*}$. Therefore, $I$ is $\mathcal{T}_{M}$-IFI $(L)$.

The following theorem provides basic properties of intuitionistic fuzzy ideals on a lattice.

Proposition 4.1. Let $L$ be a lattice. If $A$ is a $\mathcal{T}-I F I(L)$, then $[A]$ and $\langle A\rangle$ are $\mathcal{T}$-IFIs of $L$.

Proof. Assume that $A$ is an $\mathcal{T}$ - $I F I(L)$. Then for any $x, y \in L: \mathcal{T}(A(x), A(y)) \leqslant L^{*}$ $A(x \vee y)$.

Then, $\left(T\left(\mu_{A}(x), \mu_{A}(y)\right), S\left(\nu_{A}(x), \nu_{A}(y)\right)\right) \leqslant L_{L^{*}}\left(\mu_{A}(x \vee y), \nu_{A}(x \vee y)\right)$.

Hence, $\left\{\begin{array}{l}T\left(\mu_{A}(x), \mu_{A}(y)\right) \leq \mu_{A}(x \vee y), \\ S\left(\nu_{A}(x), \nu_{A}(y)\right) \geq \nu_{A}(x \vee y) .\end{array}\right.$

So, $T\left(\mu_{A}(x), \mu_{A}(y)\right) \leq \mu_{A}(x \vee y)$.

This implies, $1-T\left(\mu_{A}(x), \mu_{A}(y)\right) \geq 1-\mu_{A}(x \vee y)$

Since, $S\left(1-\mu_{A}(x), 1-\mu_{A}(y)\right) \geq 1-\mu_{A}(x \vee y)$.

Finally, $S\left(\mu_{A}^{c}(x), \mu_{A}^{c}(y)\right) \geq \mu_{A}^{c}(x \vee y)$

Then

$$
\begin{aligned}
\mathcal{T}([A](x),[A](y))= & \mathcal{T}\left(\left(\mu_{A}(x), \mu_{A}^{c}(x)\right),\left(\mu_{A}(y), \mu_{A}^{c}(y)\right)\right), \\
= & \left(T\left(\mu_{A}(x), \mu_{A}(y)\right), S\left(\mu_{A}^{c}(x), \mu_{A}^{c}(y)\right)\right), \\
\leqslant_{L^{*}} & \left(\mu_{A}(x \vee y), \mu_{A}^{c}(x \vee y)\right), \\
= & {[A](x \vee y) }
\end{aligned}
$$

Now, for any $x, y \in L: \mathcal{S}(A(x), A(y)) \leqslant L^{*} A(x \wedge y)$

Then, $\left(S\left(\mu_{A}(x), \mu_{A}(y)\right), T\left(\nu_{A}(x), \nu_{A}(y)\right)\right) \leqslant L_{L^{*}}\left(\mu_{A}(x \wedge y), \nu_{A}(x \wedge y)\right)$

Hence, $\left\{\begin{array}{l}S\left(\mu_{A}(x), \mu_{A}(y)\right) \leq \mu_{A}(x \wedge y) \\ T\left(\nu_{A}(x), \nu_{A}(y)\right) \geq \nu_{A}(x \wedge y)\end{array}\right.$

So, $S\left(\mu_{A}(x), \mu_{A}(y)\right) \leq \mu_{A}(x \wedge y)$.

This implies, $1-S\left(\mu_{A}(x), \mu_{A}(y)\right) \geq 1-\mu_{A}(x \wedge y)$

Since, $T\left(1-\mu_{A}(x), 1-\mu_{A}(y)\right) \geq 1-\mu_{A}(x \wedge y)$

Finally, $T\left(\mu_{A}^{c}(x), \mu_{A}^{c}(y)\right) \geq \mu_{A}^{c}(x \wedge y)$

Then

$$
\begin{aligned}
\mathcal{S}([A](x),[A](y)) & =\mathcal{S}\left(\left(\mu_{A}(x), \mu_{A}^{c}(x)\right),\left(\mu_{A}(y), \mu_{A}^{c}(y)\right)\right), \\
& =\left(S\left(\mu_{A}(x), \mu_{A}(y)\right), T\left(\mu_{A}^{c}(x), \mu_{A}^{c}(y)\right)\right), \\
& \leqslant L^{*} \quad\left(\mu_{A}(x \wedge y), \mu_{A}^{c}(x \wedge y)\right), \\
& =[A](x \wedge y) .
\end{aligned}
$$

Hence $[A]$ is an $\mathcal{T}$-IFI of $L$. A similar proof for $\langle A\rangle$ is an $\mathcal{T}$ - $I F I$. 
The following proposition shows that the support of a $\mathcal{T}-I F I(L)$ is an ideal in this lattice.

Proposition 4.2. Let $L$ be a lattice. The following holds

If $I$ is a $\mathcal{T}$-IFI $(L)$, then $\operatorname{Supp}(I)$ is an ideal in $L$.

Proof. Let $L$ be a lattice. Suppose that $I$ is a $\mathcal{T}-I F I(L)$ and show that $\operatorname{Supp}(I)$ is a crisp ideal in $L$.

Let $x \in \operatorname{Supp}(I)$ and $y \leq x$, then it hold that $x \vee y=x$ and $0_{L^{*}}<_{L^{*}} I(x)=I(x \vee y)$. Thus $0_{L^{*}}<_{L^{*}} \mathcal{T}(I(x), I(y))$. Using Propriety 2.1. We obtain, $0_{L^{*}}<_{L^{*}} I(y)$ hence $y \in \operatorname{Supp}(I)$.

For $x, y \in \operatorname{Supp}(I), 0_{L^{*}}<_{L^{*}} I(x)$ and $0_{L^{*}}<_{L^{*}} I(y)$. Then $0_{L^{*}}<_{L^{*}} \mathcal{T}(I(x), I(y))$. Using Propriety 2.1, then it follows from Theorem 4.3 that $0_{L^{*}}<_{L^{*}} I(x \vee y)$ hence $x \vee y \in \operatorname{Supp}(I)$. Thus, $\operatorname{Supp}(I)$ is an ideal on $L$.

Remark 4.4. The converse of Lemma 4.2 does not hold in general. Indeed, consider the lattice $L$ given in Example 3.1 and $I \in I F S(L)$ given by $I(1)=(0.6,0), I(2)=$ $(0.5,0.4), I(3)=(0.5,0.2), I(6)=(0.8,0.1)$. It is easy to verify that $\operatorname{Supp}(I)=L$ is an ideal on $L$, but $I$ is not a $\mathcal{T}-I F I(L)$.

Theorem 4.4. The $\mathcal{T}$-intersection of two $\mathcal{T}$-intuitionistic fuzzy ideals on a lattice $L$ is a $\mathcal{T}$-intuitionistic fuzzy ideal on the lattice $L$.

Proof. Let $I_{1}, I_{2} \in \mathcal{T}-I F I(L)$. Then,

$\left\{\mathcal{T}\left(I_{1}(x), I_{1}(y)\right) \leqslant L^{*} I_{1}(x \vee y)\right.$

$\left\{\mathcal{T}\left(I_{2}(x), I_{2}(y)\right) \leqslant L^{*} I_{2}(x \vee y)\right.$

Hence, $\mathcal{T}\left(\mathcal{T}\left(I_{1}(x), I_{1}(y)\right), \mathcal{T}\left(I_{2}(x), I_{2}(y)\right)\right) \leqslant L^{*} \mathcal{T}\left(I_{1}(x \vee y), I_{2}(x \vee y)\right)$.

Using Lemma 2.3

$\mathcal{T}\left(\mathcal{T}\left(I_{1}(x), I_{2}(x)\right), \mathcal{T}\left(I_{1}(y), I_{2}(y)\right)\right) \leqslant L^{*} \mathcal{T}\left(I_{1}(x \vee y), I_{2}(x \vee y)\right)$,

$\mathcal{T}\left(\left(I_{1} \cap I_{2}\right)(x),\left(I_{1} \cap I_{2}\right)(y)\right) \leqslant L_{L^{*}}\left(I_{1} \cap I_{2}\right)(x \vee y)$.

Then $I_{1} \cap I_{2}$ is an $\mathcal{T}$-IFI of $L$.

Remark 4.5. The union of two $\mathcal{T}$-IFIs need not be a $\mathcal{T}$-IFI.

Now, we introduce the notion of intuitionistic fuzzy filter on a lattice.

Definition 4.2. Let $\mathcal{T}$ be an intuitionistic fuzzy t-norm and $\mathcal{S}$ it's dual and let $L$ be a lattice and $F=\left\{\langle x, F(x)\rangle \mid F(x) \in L^{*}\right\}$ be an $I F S$ of $L$. F is called an intuitionistic fuzzy filter with respect to the intuitionistic fuzzy t-norm $\mathcal{T}$ iff the followings conditions hold

1. $\mathcal{T}(F(x), F(y)) \leqslant_{L^{*}} F(x \wedge y)$,

2. $\mathcal{S}(F(x), F(y)) \leqslant L^{*} F(x \vee y)$. 
The following immediate proposition shows that all result on ideals is true on filters.

Proposition 4.3. Let $(L, \leq)$ be a lattice, $\left(L^{d}, \geq\right)$ its dual lattice and $A \in \operatorname{IFS}(L)$. Then it holds that $A \in \mathcal{T}-I F I(L)$ if and only if $A \in \mathcal{T}$-IFF $\left(L^{d}\right)$ and conversely.

\section{Conclusion and open questions}

In this work, we have introduced the notion of $\mathcal{T}$-intuitionistic fuzzy sublattice by associating the conditions mentioned in the definition of intuitionistic fuzzy sublattice $[22,23]$. So a new equivalent definition is obtained which reduces the four conditions in only one. Thus, based on an intuitionistic fuzzy triangular norm, the study of intuitionistic fuzzy sublattices becomes so simple. Moreover, we extend the notion of an intuitionistic fuzzy ideal to a $\mathcal{T}$-intuitionistic fuzzy ideal w.r.t the lattice operations and we investigate their various characterizations and properties.

Future work is anticipated in multiple directions. We think it makes sense to study the notions of intuitionistic fuzzy prime ideals and intuitionistic fuzzy filters for other types of lattices based on the intuitionistic fuzzy setting.

\section{R E F E R E N C E S}

1. Y. S. Ahn and K. Hur and D. S. KIm: The lattice of intuitionistic fuzzy ideals of a ring. Journal Of Applied Mathematics And Computing. 19 (2005), 551-572.

2. N. JMAL and K. V. Thomas: Fuzzy lattices. Information Sciences. 79 (1994), 271-291.

3. C. Alsina: On non-distributive logical connectives for fuzzy sets. Busefal. 3 (1980), $18-29$.

4. K. T. Atanassov: Intuitionistic fuzzy sets, VII ITKR's Session, Sofia deposed in Central Sci. Technical Library of Bulg. Acad. of Sci. 1697/84, (1983).

5. K. T. Atanassov: On Intuitionistic Fuzzy Sets Theory. Springer, 2012.

6. B. O. Yuan and W. Wangming: Fuzzy ideals on a distributive lattice. Fuzzy Sets and Systems. 35 (1990), 231-240.

7. C. Cornelis and G. Deschrijver and E. Kerre: Implication in intuitionistic fuzzy and interval-valued fuzzy set theory: construction, classification, application. International journal of approximate reasoning. 35 (2004), 55-96.

8. G. Deschrijver and C. Cornelis and E. Kerre: Intuitionistic fuzzy connectives revisited. In: 9th International Conference on Information Processing and Management of Uncertainty in Knowledge-Based Systems, IPMU, 2002, pp. 1839-1844.

9. G. Deschrijver and E. KerRe: On the relationship between some extensions of fuzzy set theory. Fuzzy Sets and Systems. 133 (2003), 227-235.

10. G. Deschrijver and C. Cornelis and E. Kerre: On the representation of intuitionistic fuzzy t-norms and t-conorms. Notes on Intuitionistic Fuzzy Sets. 8 (2002), $1-10$.

11. D. Dubois and H. Prade: Fuzzy real algebra: Some results. Fuzzy Sets and Systems. 2 (1979), 327-348. 
12. D. Dubois and H. PrAdE: Towards fuzzy differential calculus part 1: Integration of fuzzy mappings. Fuzzy Sets and Systems. 8 (1982), 1-17.

13. D. Dubois and H. PrAde: Towards fuzzy differential calculus part 2: Integration of fuzzy mappings. Fuzzy Sets and Systems. 8 (1982), 105-116.

14. W. DUDEK: Intuitionistic fuzzy h-ideals of hemirings. WSEAS Transactions on Mathematics 5 (2006), 1315-1321.

15. J. A. Goguen: The logic of inexact concepts. Synthese. 19 (1969), 325-373.

16. K. HUR and S. Y. JANG and H. W. KANG: Intuitionistic fuzzy congruence on a lattice. Journal of Appl. Math. Computing. 18 (2005), 465-486.

17. K. Hur and S. Y. JANG and H. W. KANG: The lattice of intuitionistic fuzzy congruences. In: International Mathematical Forum. 5 (2006), 211-236.

18. E. P. Klement and R. Mesiar : Logical, algebraic, analytic, and probabilistic aspects of triangular norms. Elsevier, 2005.

19. E. P. Klement and R. Mesiar and E. PaP: Triangular Norms, Trends in Logic. Kluwer Academic Publishers, Dordrecht, 2000.

20. S. Milles and L. ZedAm and E. RAK: Characterizations of intuitionistic fuzzy ideals and filters based on lattice operations. Journal of Fuzzy Set Valued Analysis. 217 (2017), 143-159.

21. B. SCHWEIZER: Associative functions and statistical triangle inequalities. Puble. Math. Debrecen. 8 (1961), 169-186.

22. K. V. Thomas and L. S. NAIR: Quotient of ideals of an intuitionistic fuzzy lattice. Advances in Fuzzy Systems. 2010 (2010), 1-8.

23. K. V. Thomas and L. S. NAIR: Intuitionistic fuzzy sublattices and ideals. Fuzzy Information and Engineering. 3 (2011), 321-321.

24. L. A. ZADEH: Fuzzy sets. Information and Control. 8 (1965), 338-353.

Abdelaziz Amroune

Laboratory of Pure and Applied Mathematic

P. O. Box 166

Msila 28000, Algeria.

aamrounedz@yahoo.fr

Brahim Ziane

Laboratory of Pure and Applied Mathematic

P. O. Box 166

Msila 28000, Algeria.

And École Normale Supérieure de Bou saâda

Bou saâda 28001, Algeria.

brahziane@gmail.com 